\title{
Wave Sensitivities of Networks
}

\author{
JOHN W. BANDLER, MEMBER, IEEE, AND RUDOLPH E. SEVIORA, MEMBER, IEEE
}

\begin{abstract}
A theoretical foundation is presented for the efficient computation of first- and second-order sensitivities of networks with respect to network parameters in terms of wave variables. The concept of the adjoint network is used. First-order sensitivity formulas for a wide variety of elements are presented, including lumped and uniformly distributed elements, active and passive elements, and reciprocal and nonreciprocal elements. Parameters include electrical quantities, geometrical dimensions, and frequency. It is shown how gradients related to wave-based least $p$ th and minimax objective functions can be computed. A comparison with a method which avoids the need for analysis of adjoint networks is made. Applications in the computer-aided design of networks using efficient gradient minimization methods are envisaged.
\end{abstract}

\section{INTRODUCTION}

A RECENT PAPER by the authors [1] reviewed Tellegen's theorem and showed how it can be used in the derivation of the adjoint network method [2] of evaluating sensitivities of lumped [3] and distributed [4] networks in the frequency domain using voltages and currents. The advantages of the adjoint network method in terms of ease of implementation and computational efficiency in computer-aided network optimization should now be widely appreciated.

It is inconvenient, if not impossible, to work with currents and voltages for certain classes of networks. In the microwave region, for example, a wave description of networks is often preferable. It is the purpose of this paper to show how first- and second-order sensitivities and gradients, with respect to network parameters, can be evaluated directly in terms of wave variables employing the concept of the adjoint network.

A theorem for scattering variables which holds for properly chosen subnetworks is first presented. This theorem, which is proved without recourse to voltages and currents, is considered more general than similar results presented previously [5]-[7]. It forms the basis for the derivation of the methods for evaluating the sensitivities. Finally, the methods are related to a method which avoids the analysis of an adjoint network and uses only the results of an analysis of the given network [8].

Manuscript received February 4, 1971; revised May 6, 1971. This work was supported by the National Research Council of Canada under Grants A7239 and A5277. This paper is based on a paper presented at the 8th Annual Allerton Conference on Circuit and System Theory, Urbana, Ill., October 7-9, 1970.

J. W. Bandler is with the Department of Electrical Engineering, McMaster University, Hamilton, Ont., Canada.

$R$. E. Seviora is with the Department of Electrical Engineering, University of Toronto, Toronto, Ont., Canada.

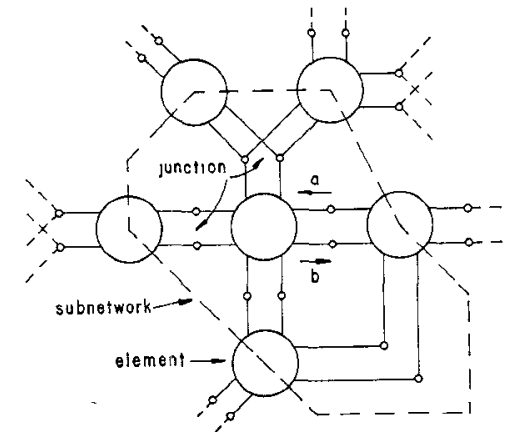

Fig. 1. Typical subnetwork.

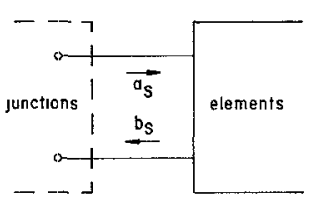

Fig. 2. Representation of incident and reflected waves within a subnetwork.

\section{A Theorem for Scattering VARIAbles}

Consider a network composed (in general) of one-port and multiport elements. Let the normalized incident and reflected waves at every port be denoted $a$ and $b$, respectively. Consider a second network also composed of one-port and multiport elements. Let the normalized incident and reflected waves at every port be denoted $\alpha$ and $\beta$, respectively. Now consider the subnetworks of each network chosen, as illustrated in Fig. 1, such that 1) the topologies of both subnetworks are the same, 2) all ports for all junctions in the subnetwork are included, and 3) corresponding ports of the two subnetworks are similarly normalized. Let

$$
a_{S}=\Gamma_{S} b_{S}
$$

and

$$
\boldsymbol{\alpha}_{S}=\boldsymbol{\Gamma}_{S} \boldsymbol{\beta}_{S}
$$

where (see Fig. 2) $a_{S}$ and $b_{S}$ contain all the incident and reflected waves in corresponding ports of the first subnetwork, $\alpha_{S}$ and $\beta_{S}$ contain the corresponding quantities in the second subnetwork, and $\boldsymbol{\Gamma}_{S}$ accounts for topology and normalization. ${ }^{1}$

1 If each junction has only two ports normalized to the same real numbers, $\Gamma$ will contain elements of value 0 or 1 . 


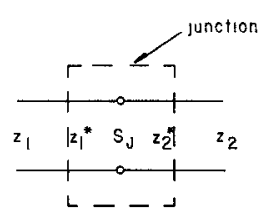

Fig. 3. Two-port junction with element normalization numbers $z_{1}$ and $z_{2}$. Note that to obtain the appropriate scattering matri : of the junction, we must assume normalization numbers $z_{1}{ }^{*}$ and $z_{2}{ }^{*}$ (see footnote 2).

Theorem

$$
\left(L_{a b} b_{S}\right)^{T} L_{\alpha \beta} \boldsymbol{\alpha}_{S}-\left(L_{a b} \boldsymbol{a}_{S}\right)^{T} L_{\alpha \beta} \boldsymbol{\beta}_{S}=0
$$

where $L_{a b}$ and $L_{\alpha \beta}$ are operators such that

$$
L_{a b} \boldsymbol{a}_{S}=L_{a b}\left(\boldsymbol{\Gamma}_{S} b_{S}\right)=\boldsymbol{\Gamma}_{S} L_{a b} b_{S}
$$

and

$$
L_{\alpha \beta} \alpha_{S}=L_{\alpha \beta}\left(\boldsymbol{\Gamma}_{S} \beta_{S}\right)=\boldsymbol{\Gamma}_{S} L_{\alpha \beta} \boldsymbol{\beta}_{S} .
$$

Proof: Assuming reciprocal junctions, $\boldsymbol{\Gamma}_{S}=\boldsymbol{\Gamma}_{S^{T}}$ so that

$$
\begin{aligned}
\left(L_{a b} \boldsymbol{b}_{S}\right)^{T} L_{\alpha \beta} \boldsymbol{\alpha}_{S}- & \left(L_{a b} \boldsymbol{a}_{S}\right)^{T} L_{\alpha \beta} \boldsymbol{3}_{S} \\
& =\left(L_{a b} b_{S}\right)^{T} L_{\alpha \beta}\left(\boldsymbol{\Gamma}_{S} \boldsymbol{\beta}_{S}\right)-\left(L_{a b}\left(\boldsymbol{\Gamma}_{S} b_{S}\right)\right)^{T} L_{\alpha \beta} \boldsymbol{\beta}_{S} \\
& =\left(L_{a b} b_{S}\right)^{T}\left(\boldsymbol{\Gamma}_{S}-\boldsymbol{\Gamma}_{S}{ }^{T}\right) L_{\alpha \beta} \boldsymbol{\beta}_{S} \\
& =0 .
\end{aligned}
$$

A special case of (3) is

$$
b_{S}^{T} \alpha_{S}-a_{S}^{T} \boldsymbol{B}_{S}=0 .
$$

\section{Discussion}

Since the theorem applies to subnetworks of the same topologies, it clearly applies to two complete networks of similar topology. Furthermore, the validity is not affected by differences in elements or element values.

Consider the subnetworks containing two-port junctions as shown in Fig. 3. $\boldsymbol{\Gamma}_{S}$ reduces to the scattering matrix of the junction, say $\boldsymbol{S}_{I}$, e.g.,

$$
\boldsymbol{\Gamma}_{S}=S_{I}=\frac{1}{z_{1}^{*}+z_{2}{ }^{*}}\left[\begin{array}{cc}
z_{2}^{*}-z_{1} & 2 \sqrt{r_{1} r_{2}} \\
2 \sqrt{r_{1} r_{2}} & z_{1}^{*}-z_{2}
\end{array}\right]
$$

where $r_{1}=\operatorname{Re}\left(z_{1}\right)$ and $r_{2}=\operatorname{Re}\left(z_{2}\right){ }^{2}$ The theorem is satisfied since $S_{J}{ }^{T}=S_{J}$. Any practical multiport waveguide junction can be considered in the same way. Since the theorem is satisfied at any junction, it is satisfied at any collection of junctions.

In the authors' opinion, the derivations presented here are more general than previous ones. Monaco and Tiberio [6], for example, considered the complete network with adjacent element ports occurring in pairs and normalized to the same real numbers. Penfield, Spence,

2 In order to satisfy the compatibility condition under complex normalization $[9$, p. 285], the scattering matrix of the junction has been written in an unconventional-looking form.

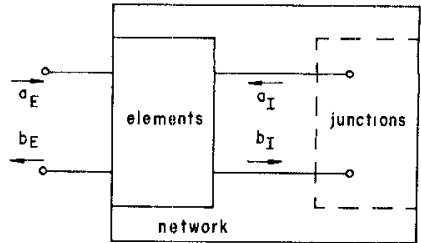

Fig. 4. Representation of a network with internal and external waves (see footnote 3 ).

and Duinker [5] used voltage and current concepts in their derivation via a generalized form of Tellegen's theorem.

\section{Corollary}

$$
\begin{aligned}
& \left(L_{a b} b_{E}\right)^{T} L_{\alpha \beta} \boldsymbol{\alpha}_{E}-\left(L_{a b} \boldsymbol{a}_{E}\right)^{T^{T}} L_{\alpha \beta} \boldsymbol{\beta}_{E}=\left(L_{a b}\left[\begin{array}{c}
\boldsymbol{b}_{E} \\
\boldsymbol{b}_{I}
\end{array}\right]\right)^{T} L_{\alpha \beta}\left[\begin{array}{l}
\boldsymbol{\alpha}_{E} \\
\boldsymbol{\alpha}_{I}
\end{array}\right] \\
& -\left(L_{a b}\left[\begin{array}{l}
a_{E} \\
a_{I}
\end{array}\right]\right)^{T} L_{\alpha \beta}\left[\begin{array}{l}
3_{E} \\
3_{I}
\end{array}\right]
\end{aligned}
$$

where (as indicated in Fig. 4) subscripts $E$ and $I$ distinguish waves associated with all external unconnected or excited ports of a subnetwork from waves associated with the remaining internal or connected ports, respectively. ${ }^{3}$ The quantities with subscript $I$ must satisfy (3). The terms on the left-hand side of (8) also appear on the right-hand side; hence (8) is valid.

\section{First-Order Sensitrvities}

\section{The Adjoint Network}

Suppose a parameter $\phi$ in a given excited network is to be varied without affecting topology. Further, let it be contained in a multiport element characterized by a scattering matrix $S$ such that

$$
b=S a \text {. }
$$

Then

$$
\frac{\partial b}{\partial \phi}=\frac{\partial S}{\partial \phi} \boldsymbol{a}+\boldsymbol{S} \frac{\partial \boldsymbol{a}}{\partial \phi} .
$$

Using (8), we may write

$$
\begin{aligned}
\frac{\partial b_{E}^{T}}{\partial \phi} \alpha_{E} & -\frac{\partial a_{E}^{T}}{\partial \phi} \beta_{E} \\
& =\left(\frac{\partial}{\partial \phi}\left[\begin{array}{l}
b_{E} \\
b_{I}
\end{array}\right]\right)^{T}\left[\begin{array}{l}
\alpha_{E} \\
\alpha_{I}
\end{array}\right]-\left(\frac{\partial}{\partial \phi}\left[\begin{array}{l}
a_{E} \\
a_{I}
\end{array}\right]\right)^{T}\left[\begin{array}{l}
\beta_{E} \\
\beta_{I}
\end{array}\right] .
\end{aligned}
$$

Note that the variables $\alpha$ and $\beta$ refer to an adjoint network. The terms relating to the element under consider-

\footnotetext{
${ }^{3}$ Quantities within the domain of $a_{E}$ and $b_{E}$ for convenience are assumed to flow across sets of two-port junctions. To facilitate this, there are no changes of normalization across any junctions which are external to the networks. Any accommodation of renormalization or multiport junctions is accomplished without loss of generality within the networks.
} 
TABLE I

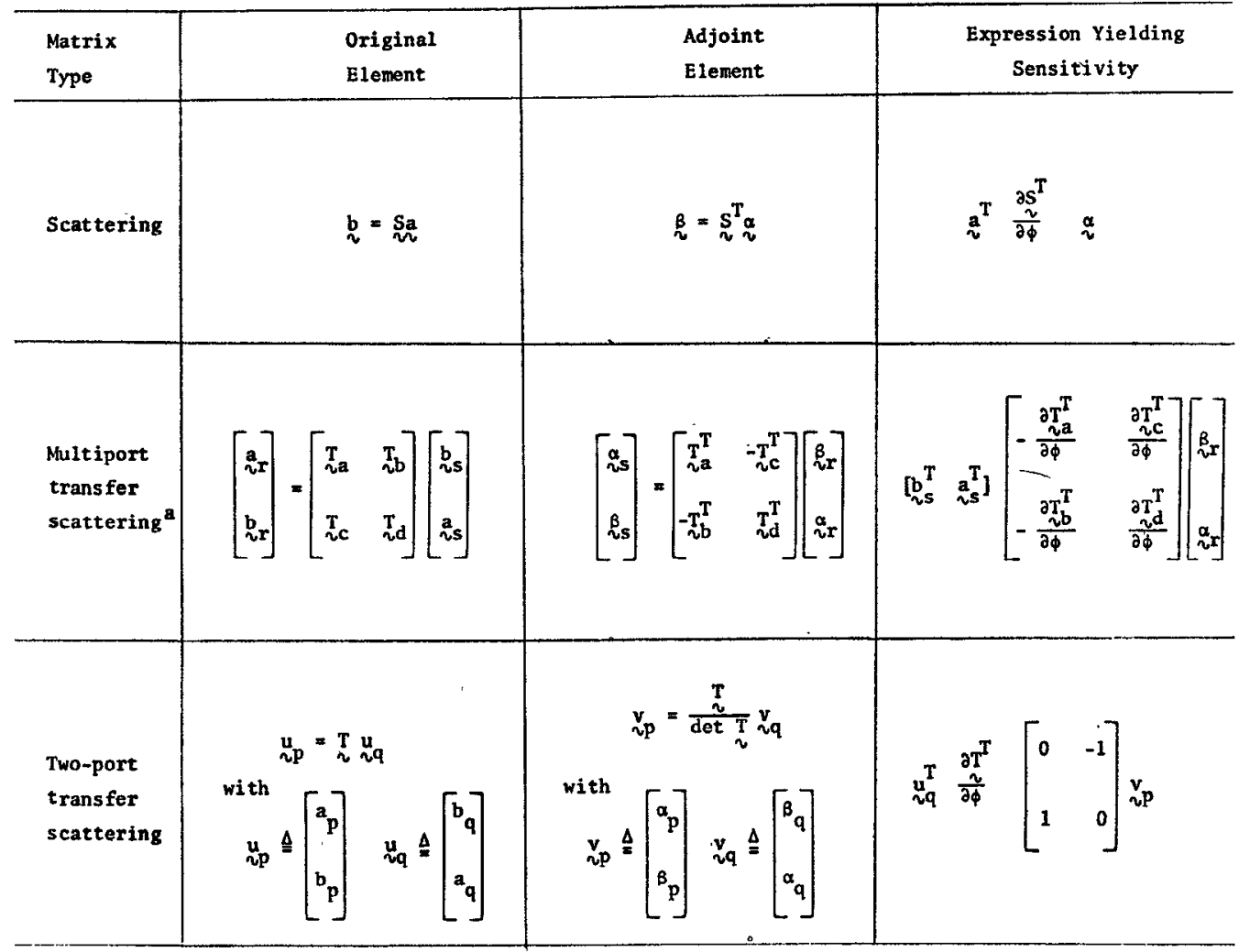

\& See footnote 5 .

ation on the right-hand side of (11) are, using (10),

$$
\boldsymbol{a}^{T} \frac{\partial \boldsymbol{S}^{T}}{\partial \phi} \boldsymbol{\alpha}+\frac{\partial \boldsymbol{a}^{T}}{\partial \phi}\left[\mathbf{S}^{T} \boldsymbol{\alpha}-\beta\right]
$$

which, defining

$$
\beta=S^{T} \alpha
$$

reduces to

$$
a^{T} \frac{\partial S^{T}}{\partial \phi} \alpha
$$

Equation (13) defines the corresponding element of the adjoint network. If all adjoint elements are similarly defined, all terms of the right-hand side of (11) except (14) drop out giving

$$
\frac{\partial b_{E}^{T}}{\partial \phi} \alpha_{E}-\frac{\partial a_{E}^{T}}{\partial \phi} \boldsymbol{\beta}_{E}=\boldsymbol{a}^{T} \frac{\partial \boldsymbol{S}^{T}}{\partial \phi} \boldsymbol{\alpha} .
$$

Assuming the network is matched (the terminations are equal to the normalization numbers), $\partial a_{E} / \partial \phi=0$ giving ${ }^{4}$

$$
\frac{\partial b_{E^{T}}^{T}}{\partial \phi} \alpha_{E}=a^{T} \frac{\partial S^{T}}{\partial \phi} \alpha=G
$$

where $G$ is a sensitivity relating to parameter $\phi$. General-

${ }^{4}$ Similar results have been derived by Monaco and Tiberio [6]. izing (16),

$$
G=\sum_{i \in E} \alpha_{i} \nabla b_{i}
$$

where $G$ is a vector of sensitivities, $\boldsymbol{\nabla}$ denotes the gradient vector, i.e.,

$$
\nabla \triangleq\left[\frac{\partial}{\partial \phi_{1}} \frac{\partial}{\partial \phi_{2}} \cdots \frac{\partial}{\partial \phi_{k_{2}}}\right]^{T}
$$

and $E$ is the set of external or excited ports.

Observe that (16) or (17) relates changes in port responses to changes in element or parameter values. Further, the elements of $G$ are obtainable from two network analyses: one of the original network and one of the suitably excited adjoint network. Table I summarizes these results and the results for other matrix descriptions of interest including the transfer scattering matrix. ${ }^{5}$ Note also that the adjoint of a reciprocal element is identical to the element itself, as is expected.

\section{Derivation of Sensitivities}

To evaluate the sensitivities, one needs to know the partial derivatives of the element scattering matrices.

5 The multiport transfer scattering matrix describes a network with an even number of ports, half of which are designated $r$ and the rest $s$. 
For many common design components these can usually be found after some manipulation. For real normalization we can frequently write [10]

$$
\frac{\partial S}{\partial \phi}= \pm \frac{1}{2 \phi}\left(1-S^{2}\right)
$$

where 1 is the unit matrix. In this case

$$
\begin{aligned}
a^{T} \frac{\partial S^{T}}{\partial \phi} \alpha & = \pm \frac{1}{2 \phi} a^{T}\left(1-S^{2}\right)^{T} \alpha \\
& = \pm \frac{1}{2 \phi}\left(a^{T} \alpha-b^{T} \beta\right)
\end{aligned}
$$

Another situation which is readily exploited is when

$$
\frac{\partial S}{\partial \phi}=-k S
$$

where $k$ is some constant. In this case

$$
\boldsymbol{a}^{T} \frac{\partial \boldsymbol{S}^{T}}{\partial \phi} \alpha=-k \boldsymbol{a}^{T} \beta
$$

Tables II and III present sensitivity expressions for a selection of components of interest with respect to useful parameters. Table III extends the results for some of the general elements of Table II to more specific elements.

\section{Gradient Computations}

Consider the design of a one-port. Suppose it is desired to optimize the reflection coefficient. With $\rho=b_{1} / a_{1}$

$$
\boldsymbol{\nabla} \rho=\frac{1}{a_{1}} \boldsymbol{\nabla} b_{1}
$$

so that, using (17), we get

$$
\boldsymbol{\nabla} \rho=\frac{1}{a_{1} \alpha_{1}} G .
$$

Consider Fig. 5. Suppose it is desired to optimize the insertion loss. Using (17) and

$$
\nabla S_{21}=\frac{1}{a_{1}} \nabla b_{2}
$$

we get

$$
\nabla S_{21}=\frac{1}{a_{1} \alpha_{2}} G .
$$

$\nabla \rho$ and $\nabla S_{21}$ are evaluated from the results of analysis of the original and adjoint networks. Any suitable analysis method will do. $\nabla \rho$ and $\nabla S_{21}$, for example, are readily used in evaluation of gradients of wave-based least $p$ th or minimax objective functions. We have already reviewed the procedure involved [1]. Here let us consider
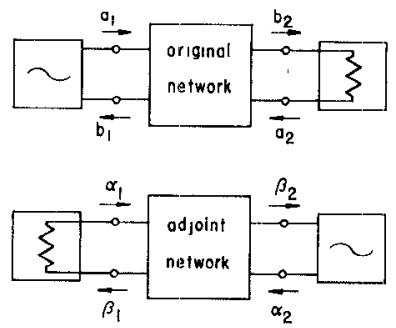

Fig. 5. Arrangement for insertion-loss design: $a_{2}=\alpha_{1}=0$.

the objective function

$$
U=\sum_{i \in E} \int_{\Omega}\left|e_{i}(\boldsymbol{\phi}, j \omega)\right|^{p} d \omega
$$

where

$$
e_{i}(\phi, j \omega) \triangleq w_{i}(\omega)\left(b_{i}(\phi, j \omega)-d_{\imath}(j \omega)\right)
$$

and where $d_{i}(j \omega)$ is a desired wave, $w_{\imath}(\omega)$ is a nonnegative real weighting function, and $\Omega$ defines the frequency range of interest. Then

$$
\begin{aligned}
\nabla \dot{U}=\sum_{i \in E} \int_{\Omega} \operatorname{Re}\left\{\left.p\left|e_{i}(\boldsymbol{\phi}, j \omega)\right|\right|^{p-w_{2}}(\omega)\right. \\
\left.\cdot \epsilon_{i}^{*}(\boldsymbol{\phi}, j \omega) \nabla b_{i}(\phi, j \omega)\right\} d \omega .
\end{aligned}
$$

Comparing (29) with (17), if we let the adjoint excitations be given by

$$
\alpha_{i}=p\left|e_{i}(\boldsymbol{\phi}, j \omega)\right|^{p-2} w_{i}(\omega) e_{i}^{*}(\boldsymbol{\phi}, j \omega)
$$

then

$$
\nabla U=\int_{\Omega} \operatorname{Re}\{G\} d \omega .
$$

The adjoint excitations are arranged to be zero at ports whose response is of no concern. Unexcited ports, of course, have zero excitations.

\section{Sensitivities With Respect to Frequency}

The foregoing results are easily extended to sensitivities with respect to frequency $[11]$. Now $\omega$ will be common, in general, to more than one element. Equation (16) must be changed to the form ${ }^{6}$

$$
\frac{\partial b_{E}{ }^{T}}{\partial \omega} \alpha_{E}=\sum_{i} a_{i}{ }^{T} \frac{\partial S_{i}{ }^{T}}{\partial \omega} \alpha_{i}
$$

where

$$
b_{i}=S_{i} a_{i}
$$

for some ith element. Formulas for the right.-hand side of (32) have to be obtained for specific elements. For

' It is assumed that the network is so arranged that frequencydependent normalization numbers or frequency-dependent junctions do not affect the $\boldsymbol{\Gamma}_{S}$ of (4) and (5), which must be independent of frequency. 
TABLE II

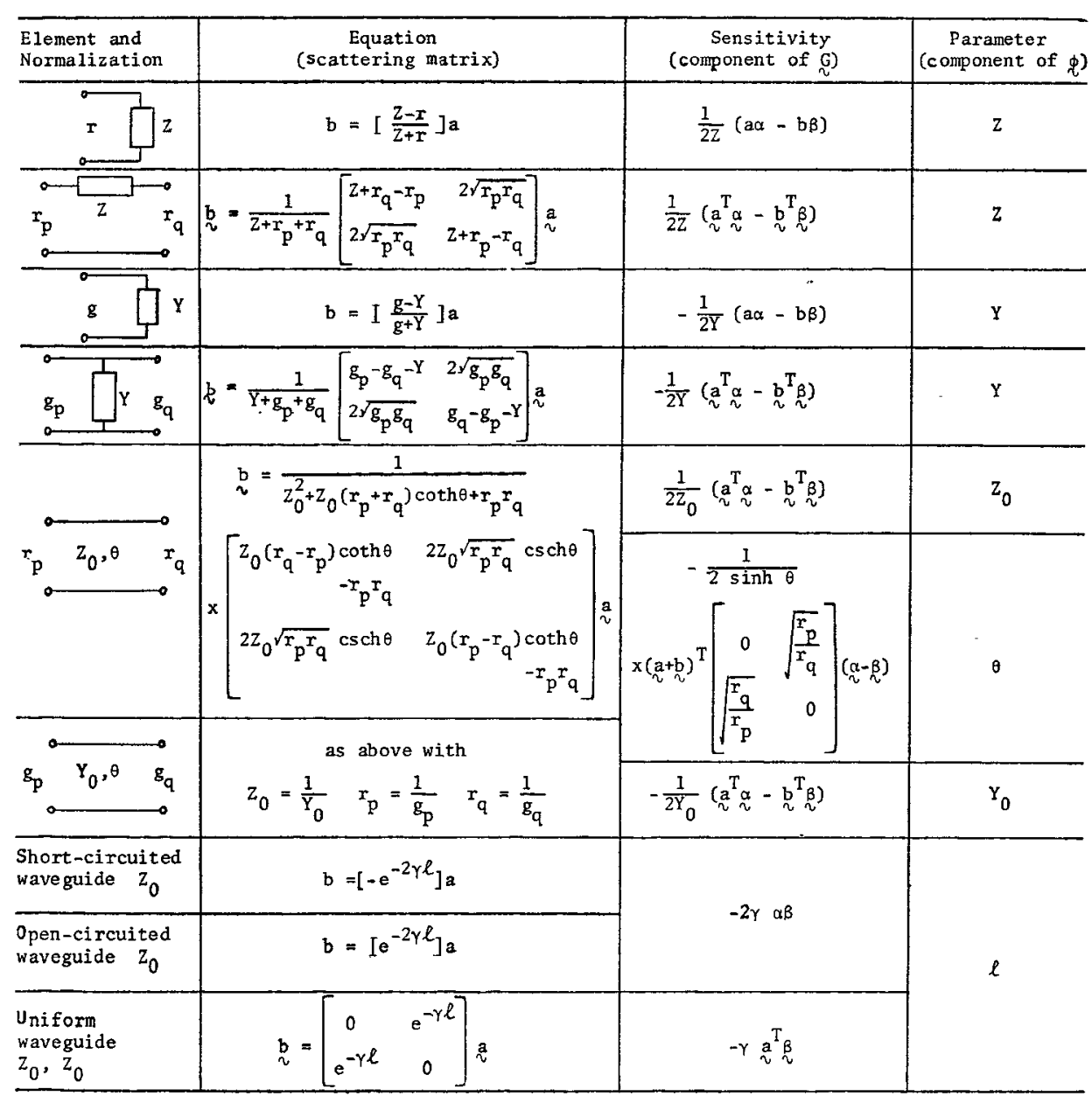

the inductor entries of Table III we would have a contribution of the form $(1 / 2 \omega) w$, for the capacitor entries $-(1 / 2 \omega) w$; for lossless transmission lines using $\beta=\omega / c$, where $c$ is the velocity of propagation, we would have $(l / c \sin 2 \beta l) w$ for the short-circuit case and $-(l / c \sin 2 \beta l) w$ for the open-circuit case, and so on. All one has to remember is if some appropriate $\partial S_{i} / \partial \chi_{j}$ are already available, then

$$
\frac{\partial \boldsymbol{S}_{i}}{\partial \omega}=\sum_{\jmath} \frac{\partial \boldsymbol{S}_{i}}{\partial \chi_{j}} \frac{\partial \chi_{j}}{\partial \omega}
$$

Thus, for example, given $\theta=j \omega l / c$, the $\omega$-sensitivity of a lossless transmission line is $j l / c$ times the $\theta$-sensitivity shown in Table II.

\section{Second-Order Sensitivities}

Consider two distinct nontopological parameters $\phi$ and $\psi$ in the original network. Assume they are contained in a multiport element with scattering matrix $S$. Applying $\partial / \partial \psi$ to $(10)$ we get

$$
\frac{\partial^{2} b}{\partial \psi \partial \phi}=\frac{\partial^{2} S}{\partial \psi \partial \phi} a+\frac{\partial S}{\partial \phi} \frac{\partial a}{\partial \psi}+\frac{\partial S}{\partial \psi} \frac{\partial a}{\partial \phi}+S \frac{\partial^{2} a}{\partial \psi \partial \phi} .
$$

Using (8), we may write

$$
\begin{aligned}
& \frac{\partial^{2} b_{E}^{T}}{\partial \psi \partial \phi} \boldsymbol{\alpha}_{E}-\frac{\partial^{2} \boldsymbol{a}_{E}{ }^{T}}{\partial \psi \partial \phi} \beta_{E} \\
& \quad=\left(\frac{\partial}{\partial \psi \partial \phi}\left[\begin{array}{l}
b_{E} \\
b_{I}
\end{array}\right]\right)^{T}\left[\begin{array}{l}
\alpha_{E} \\
\alpha_{I}
\end{array}\right]-\left(\frac{\partial}{\partial \psi \partial \phi}\left[\begin{array}{l}
a_{E} \\
\boldsymbol{a}_{I}
\end{array}\right]\right)^{T}\left[\begin{array}{l}
\beta_{E} \\
\xi_{I}
\end{array}\right] .
\end{aligned}
$$

The terms relating to the element on the right-hand side of (36) are, using (35),

$$
\begin{aligned}
\boldsymbol{\alpha}^{T} \frac{\partial^{2} S}{\partial \psi \partial \phi} \boldsymbol{a}+\boldsymbol{\alpha}^{T} \frac{\partial S}{\partial \phi} \frac{\partial \boldsymbol{a}}{\partial \psi}+\boldsymbol{\alpha}^{T} \frac{\partial S}{\partial \psi} & \frac{\partial \boldsymbol{a}}{\partial \phi} \\
& +\left(\boldsymbol{\alpha}^{T} S-\boldsymbol{\beta}^{T}\right) \frac{\partial^{2} \boldsymbol{a}}{\partial \psi \partial \phi} .
\end{aligned}
$$

Using (13), we can reduce (37) to

$$
\alpha^{T}\left(\frac{\partial^{2} S}{\partial \psi \partial \phi} \mathbf{a}+\frac{\partial S}{\partial \phi} \frac{\partial a}{\partial \psi}+\frac{\partial S}{\partial \psi} \frac{\partial a}{\partial \phi}\right)
$$

If $\phi$ and $\psi$ are identical, we have

$$
\boldsymbol{\alpha}^{T}\left(\frac{\partial^{2} S}{\partial \phi^{2}} \boldsymbol{a}+2 \frac{\partial S}{\partial \phi} \frac{\partial \boldsymbol{a}}{\partial \phi}\right)
$$


TABLE II (CONTINUED)

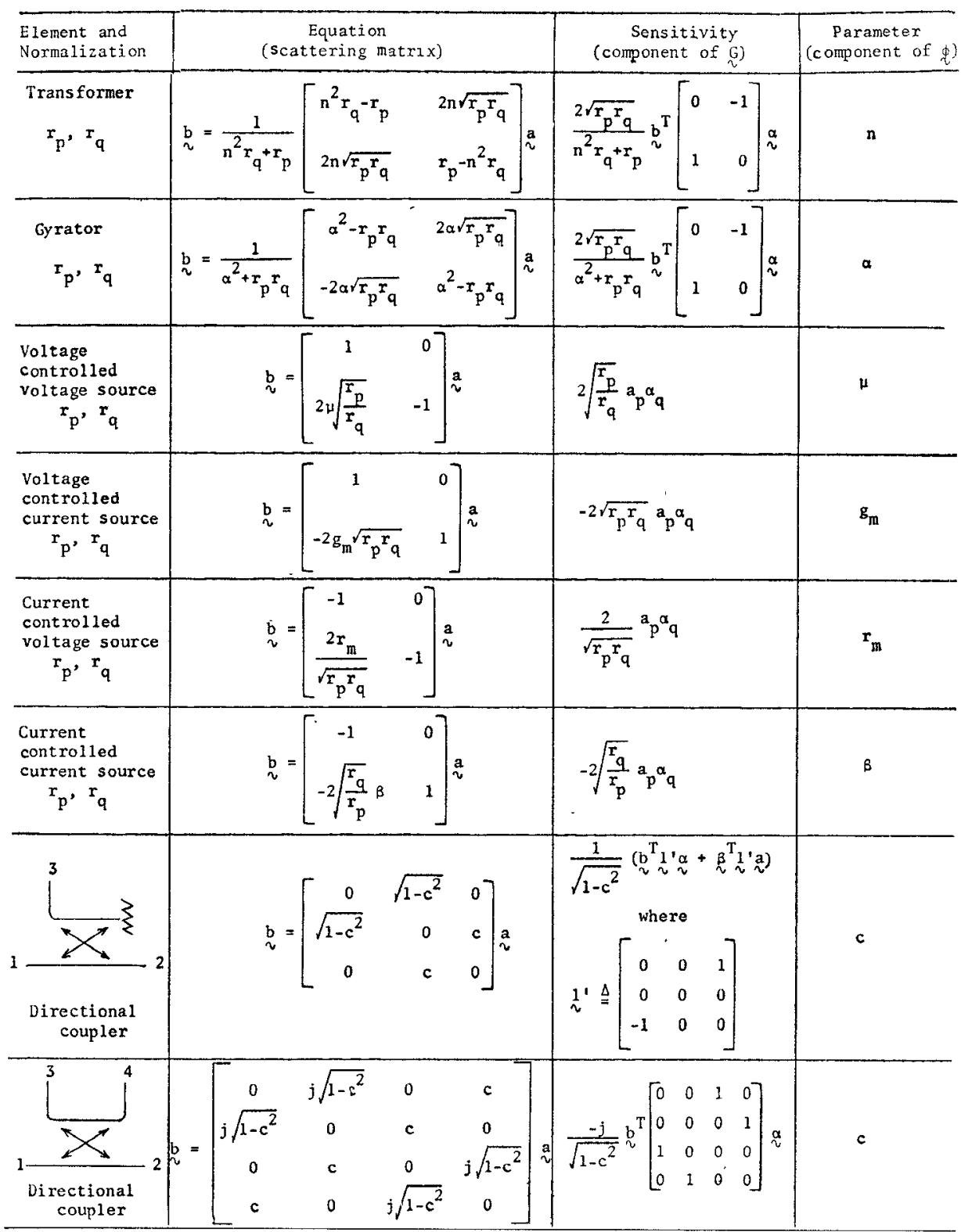

If $\phi$ belongs to an element with a scattering matrix $S_{\phi}$ and $\psi$ belongs to an element with a scattering matrix $S_{\psi}$, the corresponding expression is

$$
\alpha_{\phi}^{T} \frac{\partial S_{\phi}}{\partial \phi} \frac{\partial a_{\phi}}{\partial \psi}+\alpha_{\psi}^{T} \frac{\partial S_{\psi}}{\partial \psi} \frac{\partial a_{\psi}}{\partial \phi}
$$

where subscripts $\phi$ and $\psi$ denote quantities related to the appropriate elements.

Assuming matched terminations, $\partial^{2} a_{E} / \partial \psi \partial \phi=0$ and the left-hand side of (36) reduces to

$$
\frac{\partial^{2} b_{E}{ }^{T}}{\partial \psi \partial \phi} \alpha_{E}
$$

Summarizing the results thus far, we can write

$$
H=\sum_{i \in E} \alpha_{i} B_{i}
$$

where $H$ is a matrix of second-order sensitivities containing expressions of the form of (38), (39), or (40), as appropriate, and $B_{1}$ is the matrix of second-order derivatives of $b_{i}$, namely,

$$
B_{i}=\nabla \nabla^{T} b_{i}
$$

Equation (42) relates second-order changes in the port responses to second-order changes in the element or parameter values. The elements of $\boldsymbol{H}$ are obtainable 
TABLE III

\begin{tabular}{|c|c|c|c|}
\hline Element ${ }^{a}$ & Description & $\begin{array}{r}\text { Sensitivity } \\
\text { (component of } G \text { ) }\end{array}$ & $\begin{array}{l}\text { Parameter } \\
\text { (component } \\
\text { of } ф)\end{array}$ \\
\hline \multirow{2}{*}{ Resistor } & $Z=R$ & $\frac{1}{2 R} w$ & $\mathrm{R}$ \\
\hline & $Y=G$ & $-\frac{1}{2 G} w$ & $G$ \\
\hline \multirow{2}{*}{ Inductor } & $\because z=j \omega L$ & $\frac{1}{2 \mathrm{~L}} w$ & $\mathrm{~L}$ \\
\hline & $Y=\frac{1}{j \omega} \Gamma$ & $-\frac{1}{2 \pi} w$ & $\Gamma$ \\
\hline \multirow{2}{*}{ Capacitor } & $z=\frac{1}{j \omega} s$ & $\frac{1}{2 \mathrm{~S}} \mathrm{w}$ & $\mathrm{s}$ \\
\hline & $Y=j \omega C$ & $-\frac{1}{2 C} w$ & $\mathrm{C}$ \\
\hline \multirow{6}{*}{$\begin{array}{l}\text { Short-circuited } \\
\text { transmission } \\
\text { line }\end{array}$} & \multirow{3}{*}{$\begin{array}{l}Z=z_{0} \tanh \theta \\
Y=Y_{0} \operatorname{coth} \theta\end{array}$} & $\frac{1}{2 z_{0}} w$ & $z_{0}$ \\
\hline & & $\frac{1}{\sinh 2 \theta} w$ & $\theta$ \\
\hline & & $-\frac{1}{2 Y_{0}} w$ & $\mathrm{Y}_{0}$ \\
\hline & \multirow{3}{*}{$\begin{array}{c}Y=-j Y_{0} \cot \beta l \\
\text { (1ossless transmission } \\
\text { lines) }\end{array}$} & $\frac{1}{2 z_{0}} w$ & $z_{0}$ \\
\hline & & $\frac{\beta}{\sin 2 \beta \ell} w$ & $\ell$ \\
\hline & & $-\frac{\mathrm{T}}{2 Y_{0}} \mathrm{w}$ & $Y_{0}$ \\
\hline \multirow{6}{*}{$\begin{array}{l}\text { Open-circuited } \\
\text { transmission } \\
\text { line }\end{array}$} & \multirow{3}{*}{$\begin{array}{l}Z=Z_{0} \operatorname{coth} \theta \\
Y=Y_{0} \tanh \theta\end{array}$} & $\frac{1}{2 z_{0}} w$ & $z_{0}$ \\
\hline & & $-\frac{1}{\sinh 2 \theta} w$ & $\theta$ \\
\hline & & $-\frac{1}{2 X_{0}} w$ & $\mathrm{Y}_{0}$ \\
\hline & \multirow{3}{*}{$\begin{array}{c}Y=j Y_{0} \tan \beta l \\
\text { (loss less transmission } \\
\text { lines) }\end{array}$} & $\frac{1}{2 z_{0}} w$ & $z_{0}$ \\
\hline & & $\frac{-\beta}{\sin 2 \beta X} w$ & $\ell$ \\
\hline & & $-\frac{1}{2 Y_{0}} w$ & $\mathrm{Y}_{0}$ \\
\hline
\end{tabular}

from one analysis of the original network, one analysis of the appropriately excited adjoint network, plus as many further analyses of the adjoint network as there are ports of elements containing parameters to be varied. The reason for the further analyses is that (38), (39), and (40) require the derivatives of incident waves on these elements.

To compute these derivatives, let

$$
\beta=\beta^{\prime}+\beta^{a}
$$

represent the reflected waves from a certain element in the adjoint network, where

$$
\boldsymbol{g}^{\prime}=S^{T} \boldsymbol{\alpha}
$$

defines the element. If, in particular, we are interested in the pth port, then we set

$$
\boldsymbol{\beta}^{a}=\left[\begin{array}{llll}
1 & 0 & \cdots & 0
\end{array}\right]^{T} .
$$

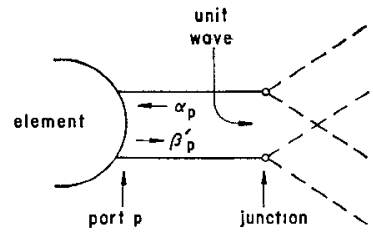

Fig. 6. Injection of a unit wave at some port $p$ in the adjoint network for evaluation of the sensitivity of $a_{p}$ in the original (see footnote 7 ).

That is, only the row corresponding to the $p$ th port is nonzero. The situation is illustrated in Fig. $6 .^{7}$

The right-hand side of (11) relating to the element [see (12)] becomes

$$
\boldsymbol{a}^{T} \frac{\partial \boldsymbol{S}^{T}}{\partial \phi} \boldsymbol{\alpha}+\frac{\partial \mathbf{a}^{T}}{\partial \phi}\left[\mathbf{S}^{T} \boldsymbol{\alpha}-\boldsymbol{\beta}^{\prime}-\boldsymbol{\beta}^{a}\right]
$$

7 The reason for making it unity is to obtain simple formulas. 
TABLE III (CONTINUED)

\begin{tabular}{|c|c|c|c|c|c|}
\hline Element ${ }^{a}$ & Description & & $\begin{array}{r}\text { Sensitivity } \\
\text { (component of } G \text { ) }\end{array}$ & & $\begin{array}{c}\text { Parameter } \\
\text { (component } \\
\text { of } \phi)\end{array}$ \\
\hline $\begin{array}{l}\text { Loss less } \\
\text { transmission } \\
\text { line }\end{array}$ & $\begin{array}{l}\text { as for transmission line } \\
\text { with real } Z_{0} \text { or } Y_{0} \\
\text { and } \theta=j \beta l\end{array}$ & $\begin{array}{l}\frac{1}{2 Z_{0}} w \\
\frac{-\beta}{2 \sin \beta C_{n}(a+b)^{T}} \\
-\frac{1}{2 Y_{0}} w\end{array}$ & $T\left[\begin{array}{cc}0 & \sqrt{\frac{r_{p}}{r_{q}}} \\
\sqrt{\frac{r_{q}}{r_{p}}} & 0\end{array}\right]_{(\substack{(\alpha-\beta) \\
\sim}}$ & & $Y_{0}$ \\
\hline $\begin{array}{l}\text { Rectangular } \\
\text { waveguide } \\
\text { operating in } \\
H_{10} \text { mode } \\
\text { (width a, } \\
\text { height b, } \\
\text { length } l \text { ) }\end{array}$ & $\begin{array}{l}\text { as for transmission line } \\
\text { with } z_{0}=b \lambda_{g} \text {, } \\
\beta \text { replaced by } \beta_{g}=2 \pi / \lambda_{g} \\
\text { where } \lambda_{g}=\lambda / \sqrt{1-(\lambda / 2 a)^{2}}\end{array}$ & $\begin{array}{l}-\frac{\lambda_{g}^{2}}{8 \mathrm{a}^{3}(a+b)^{T}}[\bar{s} \\
\frac{1}{2 b} w \\
-\frac{\beta g}{2 \sin \beta} l_{g} l_{\imath}^{(a+b)^{2}}\end{array}$ & 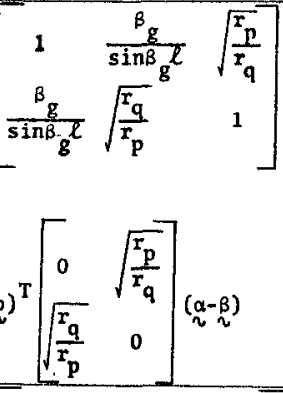 & $(\alpha-\beta)$ & b \\
\hline $\begin{array}{l}\text { Uniform } \\
\text { RC line } \\
\text { (total resistance } \\
R \text {, total } \\
\text { capacitance C) }\end{array}$ & $\begin{array}{l}\text { as for transmission line } \\
\text { with } z=\sqrt{\frac{R}{S C}} \\
\text { and } \theta=\sqrt{S R C}\end{array}$ & $\begin{array}{l}\frac{1}{4 R}\left(\sim_{\sim}^{a+b}\right)^{T} \\
-\frac{1}{4 C}(\underset{\sim}{a}+b)^{T}\end{array}$ & {$\left[\begin{array}{l}1 \\
\frac{\theta}{\sinh \theta} \sqrt{\frac{r_{q}}{r_{p}}} \frac{\theta}{\sinh \theta} \sqrt{\frac{r_{p}}{r_{q}}} \\
1 \\
\frac{\theta}{\sinh \theta} \sqrt{\frac{r_{q}}{r_{p}}}\end{array}\right.$} & $\left(\begin{array}{c}\alpha-\beta \\
\sim\end{array}\right)$ & $\mathbf{R}$ \\
\hline
\end{tabular}

a $w$ is $a \alpha-b \beta$ or $\mathbf{a}^{T} \boldsymbol{\alpha}-b^{T} \beta$ depending on whether we are dealing with a one-port or two-port element, respectively. Sensitivities for the resistor, inductor, capacitor, short-circuited, and open-circuited transmission lines are valid for these elements viewed as one-port, two-port series, and two-port shunt components.

if $\phi$ is contained in the element described by S. Using (45), (47) becomes

$$
\boldsymbol{a}^{T} \frac{\partial \mathbf{S}^{T}}{\partial \phi} \boldsymbol{\alpha}-\frac{\partial \boldsymbol{a}^{T}}{\partial \boldsymbol{\phi}} \boldsymbol{\beta}^{a} .
$$

If all external ports of the adjoint network are matched with zero excitations, $\alpha_{E}=0$, and since $\partial a_{E} /$ $\partial \phi=\mathbf{0}$, the left-hand side of (11) is zero. If all $\mathbf{3}^{a}$ are zero for the other elements, only the expression shown in (48) remains on the right-hand side of (11), and (11) reduces to

$$
\frac{\partial a_{p}}{\partial \phi}=\boldsymbol{a}^{T} \frac{\partial \boldsymbol{S}^{T}}{\partial \phi} \alpha
$$

where $\beta^{a}$ for the element is as given in (46). Clearly $\partial a_{p} / \partial \psi$, required in (38), is obtained through a similar formula. Indeed, $\partial a_{p \phi} / \partial \psi$ and $\partial a_{p \psi} / \partial \phi$, as required in $(40)$, can also be shown to have similar formulas associated with them. Thus we can obtain $\nabla a_{p}$ from one additional analysis, $\nabla a_{q}$ from another one, and so on, until all the gradient vectors necessary to produce the $\partial a / \partial \phi$ and $\partial a / \partial \psi$ for situations corresponding to (38) and (39), and $\partial a_{\phi} / \partial \psi$ and $\partial a_{\psi} / \partial \phi$ for situations corresponding to $(40)$, are available.

An alternative way of computing the elements of $H$ suggests itself. Suppose it is desired to evaluate $\boldsymbol{\alpha}_{\phi}{ }^{T}$ $\left(\partial S_{\phi} / \partial \phi\right)\left(\partial a_{\phi} / \partial \psi\right)$, as in (40), for all possible $\psi$. Assume (44) and (45), as before, but let

$$
\beta_{\phi}{ }^{a T}=\alpha_{\phi}^{T} \frac{\partial S_{\phi}}{\partial \phi} .
$$

In this case, the right-hand side of $(11)$, with $\psi$ replacing $\phi$, becomes

$$
\boldsymbol{a}_{\psi}{ }^{T} \frac{\partial \boldsymbol{S}_{\psi^{T}}}{\partial \psi} \boldsymbol{\alpha}_{\psi}-\frac{\partial \boldsymbol{a}_{\phi}{ }^{T}}{\partial \psi} \boldsymbol{\beta}_{\phi}{ }^{a}
$$

where all other $\beta^{a}$ are zero. If all external ports of the adjoint network are matched with zero excitations, $\alpha_{E}=0$, and since $\partial a_{E} / \partial \psi=0$, the left-hand side of (11), with $\psi$ replacing $\phi$, is zero. Now, from (51)

$$
\boldsymbol{\beta}_{\phi}{ }^{a T} \frac{\partial a_{\phi}}{\partial \psi}=a_{\psi}{ }^{T} \frac{\partial S_{\psi}{ }^{T}}{\partial \psi} \alpha_{\psi} .
$$

This approach, then, requires as many further analyses of the adjoint network to compute the elements of 
$H$ as there are parameters to be varied, since excitations of the form of (50) must be provided for every parameter.

All other things being equal, the choice of method for evaluating second-order sensitivities would depend on whether the number of variable parameters is less than or greater than the number of relevant ports. One could derive, for specific elements, second-order sensitivity formulas which will, of course, involve first-order expressions such as shown in Tables II and III.

\section{Relation to Direct Method}

The authors have presented a method for evaluating first- and second-order sensitivities using the results of only one analysis of the given network [8]. ${ }^{8}$ It was shown that

$$
\frac{\partial b_{E}}{\partial \phi}=\left[\begin{array}{ll}
1 & S_{E I}\left(\boldsymbol{\Gamma}-S_{I I}\right)^{-1}
\end{array}\right] \frac{\partial \boldsymbol{\Sigma}}{\partial \phi}\left[\begin{array}{c}
1 \\
\left(\boldsymbol{\Gamma}-S_{I I}\right)^{-1} S_{I E}
\end{array}\right] a_{E}
$$

where

$$
\left[\begin{array}{l}
b_{E} \\
b_{I}
\end{array}\right]=\left[\begin{array}{ll}
S_{E E} & S_{E I} \\
S_{I E} & S_{I I}
\end{array}\right]\left[\begin{array}{l}
a_{E} \\
a_{I}
\end{array}\right]=\mathbf{\Sigma}\left[\begin{array}{l}
a_{E} \\
a_{I}
\end{array}\right]
$$

and

$$
b_{I}=\Gamma a_{I} .
$$

Observe that (53) clearly leads to evaluation of the gradient vectors of the port responses independently, whereas (17) gives them only as a linear combination. Premultiply both sides of (53) by some vector $\alpha_{E}^{T}$, define the adjoint network in terms of equations corresponding to (54) and (55), and note that $\boldsymbol{\Gamma}^{T}=\boldsymbol{\Gamma}$. After some manipulation, one obtains

$$
\frac{\partial \boldsymbol{b}_{E}{ }^{T}}{\partial \boldsymbol{\phi}} \boldsymbol{\alpha}_{E}=\left[\begin{array}{c}
\boldsymbol{a}_{E} \\
\boldsymbol{a}_{I}
\end{array}\right]^{T} \frac{\partial \boldsymbol{\Sigma}^{T}}{\partial \phi}\left[\begin{array}{c}
\boldsymbol{\alpha}_{E} \\
\boldsymbol{\alpha}_{I}
\end{array}\right]
$$

To reduce the right-hand side of (56) to the right-hand side of (16), one simply notes that $\phi$ belongs to a particular element and that $\partial \boldsymbol{\Sigma}^{T} / \partial \phi$ has appropriate nonzero elements, which select out the appropriate $\alpha$ and $a$ waves.

The same kind of comparison can be carried out for second-order sensitivities, the direct-method result being

$$
\begin{aligned}
\frac{\partial^{2} b_{E}}{\partial \psi \partial \phi}= & {\left[\begin{array}{cc}
1 & S_{E I}\left(\boldsymbol{\Gamma}-\boldsymbol{S}_{I I}\right)^{-1}
\end{array}\right]\left(\frac{\partial \mathbf{\Sigma}}{\partial \psi}\left[\begin{array}{cc}
0 & 0 \\
0 & \left(\boldsymbol{\Gamma}-S_{I I}\right)^{-1}
\end{array}\right] \frac{\partial \mathbf{\Sigma}}{\partial \phi}\right.} \\
& \left.+\frac{\partial \mathbf{\Sigma}}{\partial \phi}\left[\begin{array}{cc}
0 & 0 \\
0 & \left(\boldsymbol{\Gamma}-S_{I I}\right)^{-1}
\end{array}\right] \frac{\partial \mathbf{\Sigma}}{\partial \psi}+\frac{\partial^{2} \mathbf{\Sigma}}{\partial \psi \partial \phi}\right) \\
& \cdot\left[\begin{array}{c}
1 \\
\left(\boldsymbol{\Gamma}-S_{I I}\right)^{-1} \boldsymbol{S}_{I E}
\end{array}\right] \boldsymbol{a}_{E} .
\end{aligned}
$$

${ }^{8}$ The analysis technique is based on a paper by Monaco and Tiberio [12].
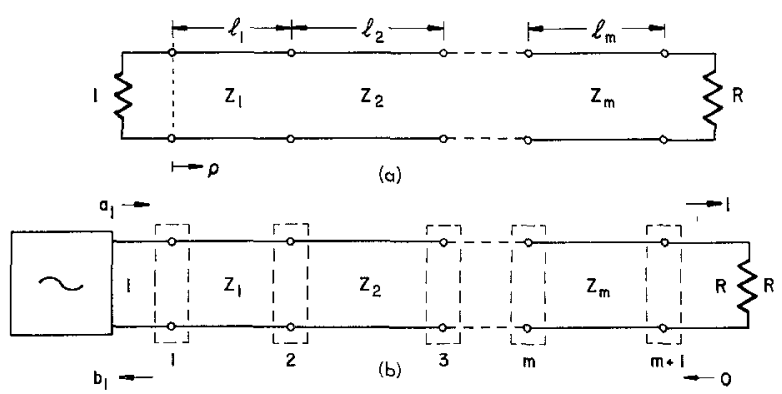

Fig. 7. (a) $m$-section resistively terminated cascade of transmission lines. (b) Arrangement for analysis indicating normalization numbers.

\section{EXAmples}

Convenient examples are provided by the $m$-section resistively terminated cascade of lossless transmission lines shown in Fig. 7(a). It is desired to compute $\boldsymbol{\nabla}|\rho(\boldsymbol{\phi}, j \omega)|$, where $\boldsymbol{\phi}$ contains the lengths and characteristic impedances of the lines. Note that

$$
\begin{aligned}
\boldsymbol{\nabla}|\rho(\boldsymbol{\phi}, j \omega)| & =\operatorname{Re}\left\{\frac{\rho^{*}(\boldsymbol{\phi}, j \omega) \nabla \rho(\boldsymbol{\phi}, j \omega)}{|\rho(\boldsymbol{\phi}, j \omega)|}\right\} \\
& =\operatorname{Re}\left\{\frac{\rho^{*}(\boldsymbol{\phi}, j \omega)}{a_{1}(j \omega) \alpha_{1}(j \omega)|\rho(\boldsymbol{\phi}, j \omega)|} G\right\}
\end{aligned}
$$

using (24). Fig. 7(b) indicates how the one necessary analysis was carried out. Thus a unit wave was assumed incident on the resistor $R$. A transfer scattering matrix analysis was carried out to find the wave $a_{1}$ necessary to produce it. The waves on both ports of the $m+1$ junctions were computed. Because of reciprocity and the fact that the normalizing numbers were conveniently $1, Z_{1}, Z_{2}, \cdots, Z_{m}$ and $R$, the formula $\left(1 / 2 Z_{0}\right)\left(\boldsymbol{a}^{T} \boldsymbol{a}-\boldsymbol{b}^{T} \boldsymbol{b}\right)$ could be used for characteristic impedances and $(-j \omega / c)$ $a^{T} b$ for lengths. In the latter case the element was treated as a transmission line normalized to its own characteristic impedance; in the former case it was treated as a transmission line with normalizations corresponding to the adjacent elements.

Optimum two- and three-section quarter-wave transformers with $R=10$ and a 100 -percent bandwidth were chosen. The parameter values were taken from [13]. Tables IV and $V$ show the components of $\nabla|\rho|$ at 0.5 $\mathrm{GHz}$ estimated from 1 - and 0.01 -percent incremental changes in the parameters compared with those obtained from the adjoint network method using one analysis. Gradient calculations at other frequencies have been made [14].

\section{Discussion}

What do we mean by a network analysis and how do we compare efficiencies of different methods of evaluating sensitivities? It is fairly obvious, for example, that evaluating just the reflection coefficient at some frequency of the network in Fig. 7 by transforming im- 
TABLE IV

Comparison of Gradient Components of the Reflection CoefferCient of the 2-Section Transformer at $0.5 \mathrm{GHz}(|\rho|=0.4286)$

\begin{tabular}{llll}
\hline $\begin{array}{l}\text { Parameter } \\
\text { Values }\end{array}$ & \multicolumn{3}{c}{ Gradient Components } \\
\cline { 2 - 5 } & $\begin{array}{l}\text { I-Percent } \\
\text { Increment }\end{array}$ & $\begin{array}{c}\text { 0.01-Percent } \\
\text { Increment }\end{array}$ & $\begin{array}{c}\text { Adjoint } \\
\text { Network }\end{array}$ \\
\hline$\ell_{1}=7.49482 \mathrm{~cm}$ & $-7.4397 \times 10^{-2}$ & $-7.3337 \times 10^{-2}$ & $-7.2326 \times 10^{-2}$ \\
$z_{1}=2.2361 \Omega$ & $-1.8250 \times 10^{-1}$ & $-1.8254 \times 10^{-1}$ & $-1.8254 \times 10^{-1}$ \\
$l_{2}=7.49482 \mathrm{~cm}$ & $-7.3745 \times 10^{-2}$ & $-7.3330 \times 10^{-2}$ & $-7.3326 \times 10^{-2}$ \\
$z_{2}=4.4721 \Omega$ & $9.0050 \times 10^{-2}$ & $9.1260 \times 10^{-2}$ & $9.1272 \times 10^{-2}$ \\
\hline
\end{tabular}

TABLE V

Comparison of Gradient Components of the Reflection COEFFICIENT OF THE 3-SECTION TRANSFORMER AT $0.5 \mathrm{GHz}(|\rho|=0.1973)$

\begin{tabular}{lrrr}
\hline $\begin{array}{l}\text { Parameter } \\
\text { Values }\end{array}$ & \multicolumn{3}{c}{ Gradient Components } \\
\cline { 2 - 5 } & \multicolumn{1}{c}{$\begin{array}{c}\text { 1-Percent } \\
\text { Increment }\end{array}$} & \multicolumn{1}{c}{$\begin{array}{c}\text { Ol-Percent } \\
\text { Increment }\end{array}$} & \multicolumn{1}{c}{$\begin{array}{c}\text { Adjoint } \\
\text { Network }\end{array}$} \\
\hline$\ell_{1}=7.49482 \mathrm{~cm}$ & $-4.4498 \times 10^{-2}$ & $-4.3777 \times 10^{-2}$ & $-4.3770 \times 10^{-2}$ \\
$z_{1}=1.63471 \Omega$ & $-4.3461 \times 10^{-1}$ & $-4.3555 \times 10^{-1}$ & $-4.3556 \times 10^{-1}$ \\
$\ell_{2}=7.49482 \mathrm{~cm}$ & $-9.1695 \times 10^{-2}$ & $-9.1294 \times 10^{-2}$ & $-9.1289 \times 10^{-2}$ \\
$z_{2}=3.16228 \Omega$ & $-6.7 \times 10^{-4}$ & $-6.5 \times 10^{-6}$ & $4.0 \times 10^{-7}$ \\
$\ell_{3}=7.49482 \mathrm{~cm}$ & $-4.3545 \times 10^{-2}$ & $-4.3767 \times 10^{-2}$ & $-4.3770 \times 10^{-2}$ \\
$z_{3}=6.11729 \Omega$ & $1.1543 \times 10^{-1}$ & $1.1638 \times 10^{-1}$ & $1.1639 \times 10^{-1}$ \\
\hline
\end{tabular}

pedances can be faster than evaluating all the necessary incident and reflected waves which will lead to the firstorder sensitivities. It would also appear, for example, that using (57) in evaluating second-order sensitivities involving only one matrix inversion might be more efficient than using the adjoint network concept.

It is hard to answer these questions, in general, without reference to particular networks and without considering whether the sparseness of the matrices will be exploited. One can certainly expect some tradeoff between complexity of the formulas, computation time, and numerical accuracy. If a given network, for example, is divided into a large number of simple subnetworks (elements), the formulas for the adjoint network method will be simple, but there will be many waves to be evaluated. If the network is divided into a smaller number of subnetworks, the formulas become more complicated but fewer waves need to be evaluated. The parameters in both cases are assumed identical.

\section{Conclusions}

This paper has presented some theoretical work which facilitates the evaluation of first- and second-order sensitivities of reciprocal and nonreciprocal networks in terms of wave variables in a systematic and straightforward manner. Applications in accurate and efficient gradient computation of wave-based objective functions for optimal design of networks by computer are envisaged. The results are not limited to microwave networks but apply to any networks for which a wave description is to be employed.

\section{ACKNOWLEDGMENT}

The authors wish to thank Dr. M. Sablatash, Department of Electrical Engineering, University of Toronto, for his cooperation and A. Lee-Chan, McMaster Data Processing and Computing Centre, McMaster University, for checking the numerical results.

\section{REFERENCES}

[1] J. W. Bandler and R. E. Seviora, "Current trends in network optimization," IEEE Trans. Microwave Theory Tech., vol. MTT18 , Dec. 1970 , pp. $1159-1170$.

[2] S. W. Director and R. A. Rohrer, "The generalized adjoint network and network sensitivities," IEEE Trans. Circuit Theory, vol. CT-16, Aug. 1969 , pp. 318-323.

[3] - "Automated network design-the frequency-domain case," IEEE Trans. Circuat Theory, vol. CT-16, Aug. 1969, pp. 330-337.

[4] J. W. Bandler and R. E. Seviora, "Computation of sensitivities for noncommensurate networks," IEEE Trans. Circuit Theory (Corresp.), vol. CT-18, Jan. 1971, pp. 174-178.

[5] P. Penfield, Jr., R. Spence, and S. Duinker, "A generalized form of Tellegen's theorem," IEEE Trans. Circuit Theory, vol. CT-17, Aug. 1970 , pp. $302-305$.

[6] V. A. Monaco and P. Tiberio, "On linear network scattering matrix sensitivity," Alta Freq., vol. 39, Feb. 1970, pp. 193-195.

[7] J. W. Bandler and R. E. Seviora, "Sensitivities in terms of wave variables," in Proc. 8th Annu. Allerton Conf. Circuit and System Theory, Oct. 1970, pp. 379-387.

[8] _- "Direct method for evaluating scattering-matrix sensitivities," Electron. Lett., vol. 6, Nov. 1970, pp. 773-774.

[9] E.S. Kuh and R. A. Rohrer, Theory of Linear Active Networks. San Francisco, Calif.: Holden-Day, 1967.

[10] M. Sablatash and R. Seviora, "Sensitivity invariants for scattering matrices," IEEE Trans. Circuit Theory (Corresp.), vol. CT18, Mar. 1971, pp. 282-284.

[11] G. C. Temes, "Exact computation of group delay and its sensitivities using adjoint-networls concept," Electron. Lett., vol. 6, July 1970 , pp. $483-485$.

[12] V. A. Monaco and P. Tiberio, "Automatic scattering matrix computation of microwave circuits," Alta Freq., vol, 39, Feb. 1970, pp. $165-170$.

[13] J. W. Bandler and P. A. Macdonald, "Optimization of microwave networks by razor search," IEEE Trans. Microwave Theory Tech., vol. MTT-17, Aug. 1969, pp. 552-562.

[14] J. W. Bandler, "Conditions for a minimax optimum," IEEE Trans. Circuit Theory (Corresp.), vol. CT-18, July 1971, pp. 476479. 